\title{
Sensor-Cloud: A Hybrid Framework for Remote Patient Monitoring
}

\author{
Subasish Mohapatra, \\ Dept of CSE, ITER, \\ Siksha O Anusandhan University
}

\author{
K. Smruti Rekha, \\ Dept of CSE, ITER \\ Siksha O Anusandhan University
}

\begin{abstract}
Remote patient monitoring is a crucial/decisive issue in healthcare system. Healthcare systems strive to improve the quality of service and cost effectiveness of care. Due to the distributed computing nature of wireless sensor network, they play an important role in providing solution to healthcare obstacles based on real time data. Sensor networks are responsible for integrating the physical world with digital world. But sensors are constrained with insufficient computational power, less storage resources, limited lifetime \& prone to failure. On the other hand cloud is full of huge memory, high band width, etc which swifts the data processing faster and provide quick response to user. In this paper we have proposed a hybrid frame work by integrating sensor network with cloud computing to monitor the remote patient health status and take necessary aid.
\end{abstract}

\section{Key Words}

Sensor network, cloud computing, sensor cloud architecture, Patient Monitoring.

\section{INTRODUCTION}

Leveraging towards new technologies has a great impact on worldwide healthcare system. First and foremost applications enabled mobile users to send and check email, browse websites, play games, and other activities that were done via desktop or laptop computers [1] [2]. Other device-specific applications that allowed users to do everything from tracking and controlling financial information to monitoring caloric intake and arming security systems in their homes from remote locations [3]. Commercially, mobile devices changed how companies inventoried and monitored their products, how shipping companies scanned and reported package pickups, and even how meal orders in some restaurants were recorded and delivered to the kitchen staff [4][5]. More sophisticated devices emerged that enabled government and military authorities to monitor and track persons domestically and on the battlefield [6]. Vehicles can able to guide drivers to their destinations, avoid collisions with other vehicles, and sense road conditions, among other advantages [7].

\section{Application in Remote Healthcare}

Modern technology is gradually shifted from client server based model to cloud based platform [22]. Present cloud based mobile technology allows the physicians, doctors and patients to share information inside hospital environment [8]. It concentrates on collection of data from patients during a period of time they are often suffering the most-after initial post operative releases from hospital/clinics [22]. Continuous monitoring of patient's health status, storing the updated information and transmitting the records require high end system [9]. Wearable devices have been developed to monitor glucose levels in diabetic patients and heart bit rate in cardiology patients [10]. Now telemedicine now allow patients in remote areas to have x-rays, MRIs and other diagnostic tests closer to their homes [11]. The general public and physicians with the help of telemedicine can access informative websites such as WebMD to obtain information about specific medications and their interactions [12]. In healthcare system, much focus has been placed on making the work of physicians as well as medical services more efficient with automated processes using modern hardware and software techniques. Handling real time problems for elderly patients and tracking real time data for post operative patients are vital issues in hospital system. The ultimate goal is to increase the availability of medical care in order to reduce the demands on hospital service Proposed system reduces the time of routine check-up. It also allows emergency situations to be handled immediately [13].

Betterments in electro- mechanical devices have led to creation of sensor nodes capable of sensing and communicating one or more vital physiological signals from patient and integrating them into a sink for health monitoring. Instead of attaching sensors to medical equipments, wearable sensors like accelometers, gyroscpe, smart pills, etc are attached to human body internally or externally which continuously monitor the blood pressure rate, heartbeat, fall detection, etc. Sensors are deployed in home and hospital environment for monitoring and collecting real time data. These sensor nodes help in continuous health monitoring of post operative patients at hospital and elderly patient at home environment with almost real time updates of medical records. Since cloud computing provides plenty of applications, platforms and infrastructure over internet, it may combined with sensor network in the application areas like environmental monitoring, weather forecasting, transport business, Health care, military applications etc. The integration of sensor network with cloud computing would be an exciting approach to explore among the research community. Our proposed model is used to monitor different groups of patients and to provide the information regarding their health status to the physicians, researchers. The data are published via web servers. Healthcare professionals, researchers and patients can access the long-term physiological data via the Internet. A secure cloud server allows authenticated users to access real-time patient information to consult with medical specialists located at distant places. [14]

\section{RELATED WORKS}

In order to access the real time data from remote patient different researchers have developed different models with different concept.

Sensor cloud technology enables the early detection of adverse conditions and diseases [14]. A number of recent research focuses on wearable sensors which are attached to patient's body to regulate their health condition. By the help of semi markov 
conditional random field complex activities like eating, exercising, reading can also be predicted [15]. Researchers at MIT lab have developed MIThril, a wearable computing platform compatible with both custom \& off the shelf sensors. A Harvard university project " code blue" has researched on developing WSN for medical applications which tells that when sensors are attached to human body, it transmits vital signals by the help of ad-hoc network to healthcare providers [16]. A voice enhanced service using voice over internet protocol (VoIP) is provided in order to communicate with the patients for better diagnose their problems and to provide better aid. Once the data from sensor nodes are collected they are then uploaded to the cloud gateway, the gateway classifies data into health data, gyroscope, accelerometer data, imaging data and finally store in a local database. The filtering module filters redundant and noise data to reduce communication overhead before sending to the cloud. The filtered data is also updated to the local database. Activity [18] and context are forwarded to the ontology engine for representation and inference higher level activities and context. Ontology engine is designed to deduce high-level activities and make decisions [17] according to user profile and performed activities. The current trend towards Telemedicine and Telecare evident in the UK [19] has seen an explosion in the range of locations where advanced medical care needs to be delivered. E-medicine initiatives such as NHS Direct have illustrated the need to maximize the flexibility of delivery of health care. Existing trials in Telemedicine and Telecare such as those carried out by the Oxford centre for e-health [19]. The Biomedical Informatics group at Nottingham University [20] and the Glasgow Royal Infirmary and Glasgow University [21] have demonstrated the feasibility of remotely monitoring patients as part of an overall care program or as a part of clinical trial. UbiMon proposes a platform which applies implantable sensors to get vital data. But it lacks on demand way to handle collected data [24]. Uniform access, system management and network management are the constraints of sensor grid architect true. EyeOS an open source desktop is used as cloud, but here the data is passed through a local machine through SaaS [23]. Our proposed sensor cloud architecture gives a virtualized view of managing different resources available in the cloud for uniform access . The rest of this paper is organized as follows: Section 3 explores sensor cloud architecture for patient monitoring. Section 4 describes how sensor cloud helps monitoring different kind of patients; Section 5 describes conclusion and future work towards this direction.

\section{PROPOSED SENSOR CLOUD ARCHITECTURE}

Sensors enabled patient monitoring system using cloud computing concepts monitors human health, activities and shares information among doctors, care-takers, clinics, and pharmacies in the cloud.

WSNs are deployed in home environments for monitoring and collecting raw data. Sensed data is uploaded to cloud using a fast and scalable sensor data dissemination mechanism. Sensor networks are connected with gateway through common interface in different way such as serial, USB, Bluetooth or Zigbee. Gateway receives the raw data from the communication ports and kept in a buffer for further processing. Virtualization manager helps in aggregation of heterogeneous and autonomous resources. This component is divided into five subcomponents namely Storage server, Data Processor, Medical Server, Command interpreter and Common Interface. The gateway classifies data into health data,

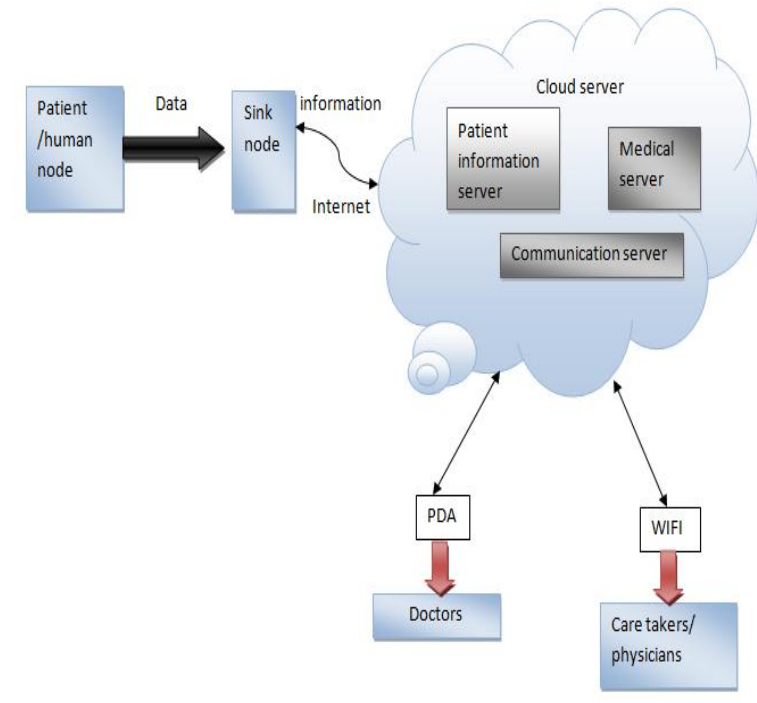

Fig 1: Sensor cloud platform for patient monitoring

Gyroscope and accelerometer data and imaging data, and store in a local buffer. Then that is transmitted to Storage server. Data processor retrieves the data from the server and processes according to its type. The filtered data is also updated to the Medical server. The filtering module filtered redundant and noise data to reduce communication overhead before sending to the cloud. Command interpreter is responsible for providing the reverse communication channel from the gateway to the Sensor Network for processing and interpreting various commands issued by different applications and generates the code that is understood by the sensor nodes. System Manager is responsible for processing and archiving the sensor data and also manages the system resources. Cloud authentication and access control is the major roles of system manager. Service Registry maintains the credentials of different consumer's applications register to publisher/subscriber system for various sensor data required. For each application, registry component stores user subscriptions, sensor data and sensor event types the application is interested in. Each application is associated with a unique application ID along with the Service Level Agreement (SLA). Stream Monitoring and Processing module is divided into three sub components i.e. registry component, analyzer component and disseminator component.

Registry Component (RC) stores user subscription of different application and user specific sensor data types of those users who register to Pub/Sub agent. It also sends all user subscriptions along with application ID to the disseminator component for event delivery. Analyzer Component (AC) analyzes the incoming sensor data or event to match with user subscriptions in the service registry. If the sensor data matches with the interest of the subscriber, the same is handed over to the disseminator component to deliver to the appropriate users. Disseminator Component (DC) receives the data or event of interest from the analyzer component and delivers the data through application interface to the subscribed applications. Query requested from a service/application, the query/response manager fetches data from the communication and sends it to the requester which is managed by communication server. Virtual Machine Manager is responsible for facilitating uniform access to multiple subscribers at the same instance. Application Specific Interface consists of two components such as Business process report and core Application for different type of users. 


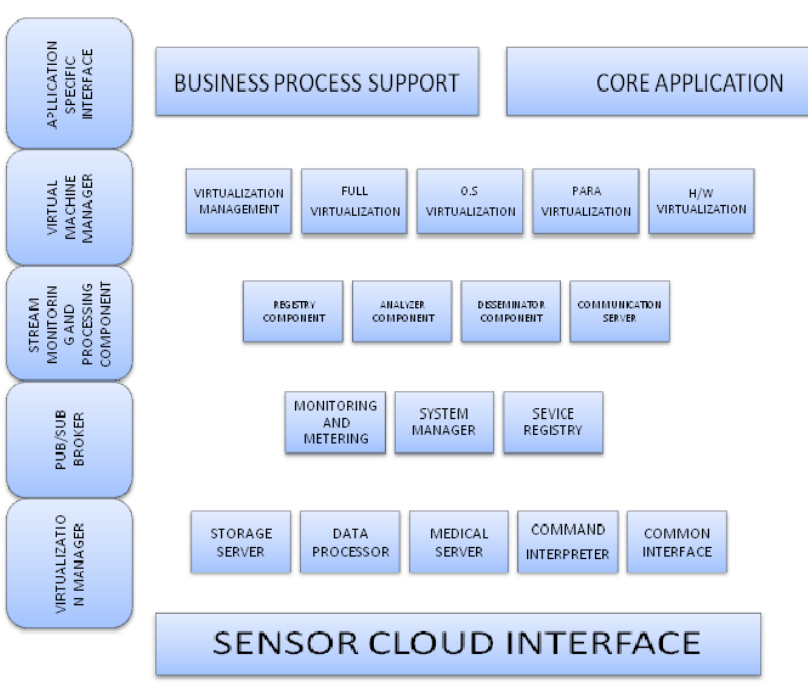

Fig 2: Sensor cloud protocol stack

\subsection{Service Authentication}

To access information about the patients the physicians need authentication. Each patient has their unique ID and password like social networks. The doctor or medical staffs have to authenticate themselves under the patients account to know their health conditions. Clouds eliminate the responsibility to install and maintain hardware and basic computational services away from the client. A cloud server is one where virtual shared servers provide hardware, a software infrastructure or an application as a service to its customers. A special type of software called middleware (e.g. ANEKA) is used to allow computers that are connected to the network to communicate with each other. This system guarantees the integrity and confidentiality of medical data.

\subsection{Servers used in Sensor cloud architecture}

Different types of servers such as Storage server, Medical server, and Communication server are used to form cloud server.

\subsubsection{Patient's information server}

Terabytes of data have to be collected during this monitoring process of elderly patients and post operative patients. Sensor devices are resource constrained in nature, with a limited processing and communication ability, and hence we are applying and adding cloud technology to store the data collected by the sensor nodes in the storage server located in their physical surroundings.

\subsubsection{Medical server}

The main purpose of the medical server is to do the on-line and off-line analysis and prediction from the gathered information. The large amount of collected physiological data will allow a quantitative analysis of various conditions and patterns. Data mining is a complex process that allows discovering unsuspected meaningful knowledge in large databases.

\subsubsection{Communication server}

Data from WSNs are stored in a communication server to facilitate analysis, diagnosis and alerting when a life-threatening event occurs. The criterion of our architecture framework design is to make the use of the cloud infrastructure completely transparent to the physicians, caretakers, and researchers. The threshold-based algorithm for detecting abnormalities in physiological signals and the fall detection algorithm for detecting abnormalities in physical activities are stored in distributed algorithm pools. When an anomaly is detected in the patient's vital sign, the application software generates an alert for the physicians, caretakers and emergency department personnel. Different types of user to access the patient information in real time.

a. Caretakers may log onto a website under a patient account to review vital statistics and the location of the elderly patients who had been tagged with sensors.

b. Medical specialists, located at a distance, may view their respective patients' real-time health status and alert the caretakers near the patients through the doctor's login.

c. Emergency personnel have full access to all data in the system. They can add new patients, health professionals, and administrators. They can access and change the information of all the participants.

\section{SENSOR CLOUD FOR PATIENT MONITORING}

Now we will discuss how sensor cloud helps in monitoring post operative and elderly patients.

\subsection{Monitoring Post Operative Patients}

It is clinically desirable to monitor the post-operative patients after surgery as they are always at a risk of post operative pains and require high degree of care by their physicians [22]. It may result in an early intervention to prevent adverse outcomes.

Wearable and implantable body sensors attached to patient's body give contextual information and alerting messages in continuous monitoring of patients at large. This wireless hospital system consists of the following components.

(i) A front end composed of the different sensors for the recording of the vital signals that are demanded by the application (ECG, respiration and activity level).

(ii) A patient station, which consists of a mote device that receives information from the sensors and is responsible for the first stage of data processing in the data communication. The received signal is sent to a central server (cloud server) for analysis.

(iii) A central server, which is the core processing element, receives the patient's data from the patient station for analysis. The analysis is done using mote view software and generates early alerts to the medical professionals, caretakers and emergency medical department personnel. 


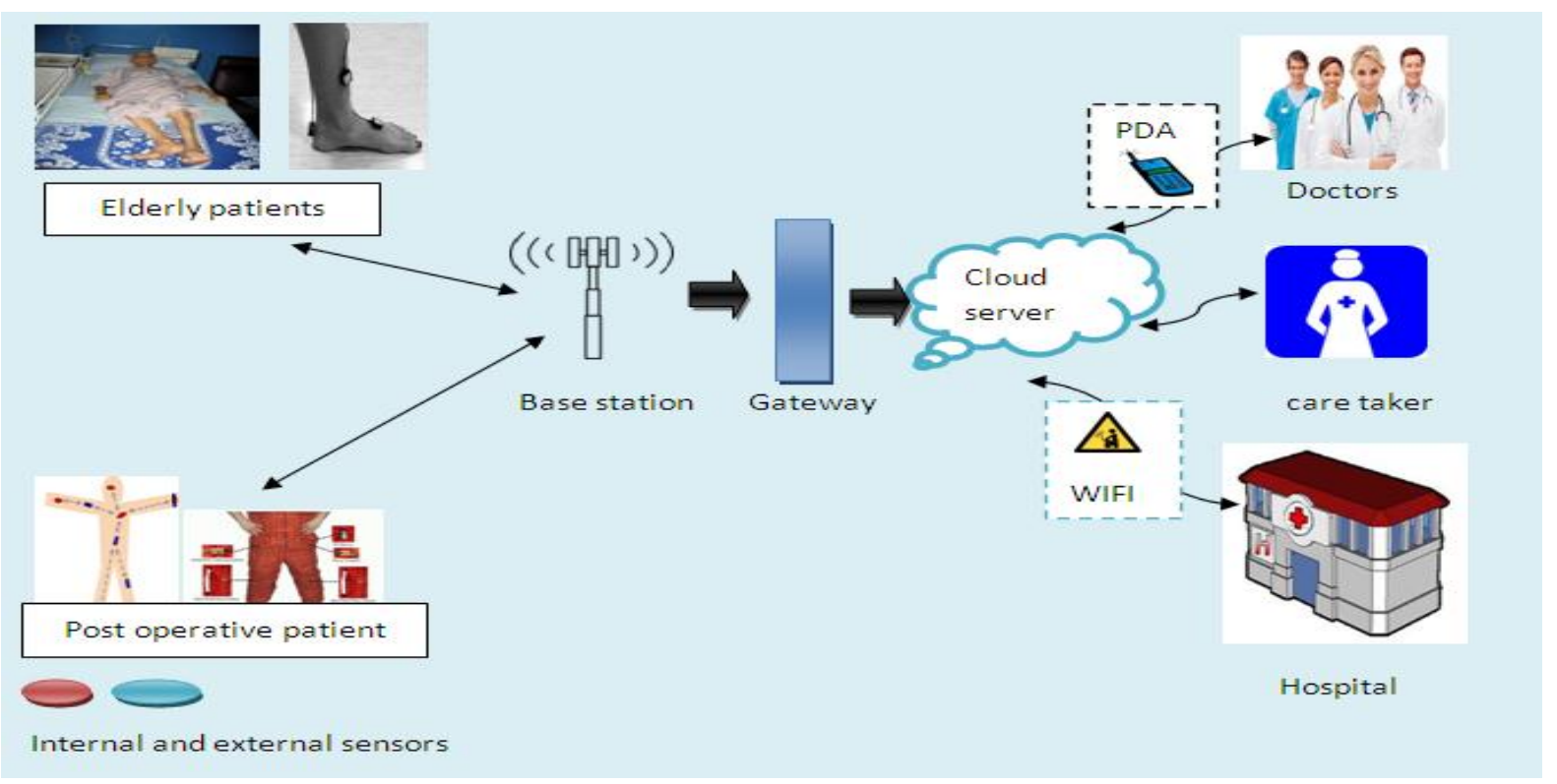

Fig3: sensor cloud serving different groups of patients

\section{Monitoring Elderly Patients}

Recently, many research groups have shown interest in applying body sensor networks for remote monitoring of elderly patient. Most homes moved to smart home which contains sensor appliances like smart computers, TVs, smart floors which contain sensor nodes in it to detect human activities, video systems. Our software offerings are homeowner friendly and can be installed on existing PCs. A wireless system could be set up throughout the home to sense physiological parameters such as ECG, heart rate, body temperature, etc., motion patterns and visual detection of elderly patients. However, a wireless home application could provide the freedom and ease of use necessary to make home health monitoring beneficial for continuous long-term monitoring as part of a diagnostic procedure for elderly patients. This system consists of the front end, patient station, and server.

The front end is composed of different types of sensor for monitoring continuous physiological parameters as well as motion pattern and visual monitoring of the elderly patients at home. We have used ECG sensors, heart rate sensors and temperature sensors for physiological monitoring, accelerometer sensors attached to the human arm to determine patients' movements and 3D cameras for visual monitoring of elderly patients. The patient station is an IRIS sensor node, a new family of ultra low-power, long-range wireless sensor network product devices that receives information from the physiological sensors. The 3D camera in the home can be connected with an Imote2 sensor node (IPR2400CA) for visual monitoring of elderly patients in home environments in addition to monitoring the physiological signals. The Imote 2 sensor node is aimed at applications involving data-rich computations, where there is a need for both high performance and high bandwidth, which require greater processing capability, and low- power operation with a low duty cycle to achieve longer battery life. Sensor nodes are responsible for sensing as well as the first stage of sensory data processing in the data communication. The received signal is sent to the central server for analysis. When an anomaly is detected, the mote view software installed in the cloud server generates an alert to the doctors and caretakers via a Personal
Data Assistant (PDA) or cell phones. The above architecture shows how sensor cloud serves different group of patients.

\section{BENEFITS}

I. Access your real time data at all time.

II. A physical storage centre is no longer needed.

III. Easily scalable, so users can add or subtract storage based on their own needs.

IV. More bandwidth, more processing power, more secure aspects, etc.

\section{CONCLUSION}

Sensors enabled patient monitoring system using cloud computing monitor human health and share the information among doctors, care-takers, researchers, and pharmacies from the Cloud to provide low-cost and high-quality care to users. WSNs are deployed in home environments for monitoring and collecting raw data. Future enhancements such as latest sensor devices and several other sensor devices can be deployed using a much more efficient microprocessor kit, and cloud security can be increased consistently. Other aspects such as authorization, authentication and user management must be incorporated in the framework. Different policy management should be implemented in order to enhance the privacy and security.

\section{REFERENCES}

[1] K. Hameed, "The Application of Mobile Computing and Technology to Health Care Services." Telematics and Informatics 20.2 (2003) pp. 99-106.

[2] Dick. Friedman, "Mobile Computing." Electrical Wholesaling. April1,2006.http://ewweb.com/software.

[3] O'Mara, L. Deborah "Data Streams \& Digital Dreams." Security Dealer \& Integrator. 2010. http://www.securityinfowatch.com/ 
[4] "Two So. Calif. eateries add mobile order processing tools." Nation's Restaurant News. December 17, 2007. http://www.nrn.com/article.

[5] Cindy. Waxer, "How UPS Is Scanning and Printing Its Way to Savings" http://www.cio.com/article/498056.

[6] Courtney. Howard, "Warfighters on the Digital Battlefield Require Robust Information Technology for Secure, Reliable, Real-time Access to Mission-critical Information." Military \& Aerospace Electronics. June 16, 2010. http://www.militaryaerospace.com.

[7] T. Umedo, , T. Higashino, C. K. Toh, and T. Isu. "An Intervehicular-Communication Protocol for Distributed Detection of Dangerous Vehicles." IEEE Transactions on Vehicular Technology 59.2 (2010): 627.

[8] Eileen Colkin Cuneo. "Mobile Care." InformationWeek. 2004. Online at http://www.informationweek.com/news/

[9] Lang, Kathleen. "MedQuist to Showcase Next Generation of Mobile Web Dictation at HIMSS 2008." MedQuist. Online at http://www.medquist.com/Portals.

[10] E.Renard, "Implantable Glucose Sensors for Diabetes Monitoring." Minimally Invasive Therapy \& Allied Technologies 13.2 (2004): pp.78-86.

[11] Ganapathy, Krishnan, and Aditi Ravindra. "Telemedicine in India: the Apollo Story."Telemedicine and E-health, Volume: 15 Issue 6: August 6, 2009

[12] "WebMD Launches New Version of Free Mobile Application for Physicians". Lymberis, Smart wearables for remote health monitoring, from prevention to rehabilitation: Current R\&D, future challenges, in: Proceedings of 4th International IEEE EMBS Special Topic Conference on Information Technology. Applications in Biomedicine, 2003, pp. 272-275.

[13] M. Pallikonda Rajasekaran, S. Radhakrishnan, P. Subbaraj, Remote patient monitoring system using wireless sensor networks, International Journal of Healthcare Technology and Management 9 (3) (2008) pp.247_257.

[14] Alexandros Pantelopoulos and Nikolaos G. Bourbakis, "A Survey on Wearable Sensor-Based Systems for Health
Monitoring and Prognosis", IEEE Trans. on Systems, Man, and Cybernetics- PART C: Applications and Reviews, USA, Volume- 40, No- 1, January 2010, PP. 4- 10.

[15] Y-T. Zhang, X.Y. Xiang, C.C.Y. Poon, The evaluation of nodes of body sensor networks: Wearable blood pressure measuring devices, in: Proceedings of the International Workshop on Wearable and Implantable Body Sensor Networks, 2006.

[16] Asad Masood Khattak, La The Vinh, Dang Viet Hung, Phan Tran Ho Truc, Le Xuan Hung, D. Guan, Zeeshan Pervez, Manhyung Han, Sungyoung Lee, and Young-Koo Lee, "Context- aware Human Activity Recognition and Decision Making”, IEEE HealthCom, Korea, 2010, PP. 113- 117.

[17] Asad Masood Khattak, Zeeshan Pervez, Koo Kyo Ho, Sungyoung Lee, Young- Koo Lee, "Intelligent Manipulation of Human Activities using cloud computing for u- Life Care", IEEE Annual Int. Symposium on Applications and the Internet, Korea, 2010.

[18] The oxford centre for e-health. http://www.medicine.ox.ac.uk.

[19] The biomedical informatics group at Nottigham University. http://www.eee.nott.ac.uk/medical/.

[20] Computer assisted reporting of electrocardiograms, Glasgow University.Http://www.gla.ac.uk/departments.

[21] " How are you feeling? A social network model to monitor the health of post operative patients." James J. Mulcahy, Shilong Huang, Junwei Cao and Fan Zhang.

[22] J.Thirunavukkarasu, Dr. N.Kumaratharan."Sensor enabled patient monitoring system using cloud computing concepts" vol.2 issue 2, feb.2012, pp.247-252.

[23] Carlos Oberdan Rolim, Fernando Luiz Koch, Carlos Becker Westphall, Jorge Werner, Armando Fracalossi, Giovanni Schmitt Salvador, "A Cloud Computing Solution for Patient's Data Collection in Health Care Institutions." 